\title{
A!
}

This is an electronic reprint of the original article.

This reprint may differ from the original in pagination and typographic detail.

Bică, M.; Koivunen, V.

\section{Multicarrier Radar-communications Waveform Design for RF Convergence and Coexistence}

Published in:

44th IEEE International Conference on Acoustics, Speech, and Signal Processing, ICASSP 2019; Brighton; United Kingdom; 12-17 May 2019 : Proceedings

DOI:

10.1109/ICASSP.2019.8683655

Published: 01/05/2019

Document Version

Peer reviewed version

Please cite the original version:

Bic, M., \& Koivunen, V. (2019). Multicarrier Radar-communications Waveform Design for RF Convergence and Coexistence. In 44th IEEE International Conference on Acoustics, Speech, and Signal Processing, ICASSP 2019; Brighton; United Kingdom; 12-17 May 2019 : Proceedings (pp. 7780-7784). [8683655] (Proceedings of the IEEE International Conference on Acoustics, Speech, and Signal Processing). IEEE. https://doi.org/10.1109/ICASSP.2019.8683655

This material is protected by copyright and other intellectual property rights, and duplication or sale of all or part of any of the repository collections is not permitted, except that material may be duplicated by you for your research use or educational purposes in electronic or print form. You must obtain permission for any other use. Electronic or print copies may not be offered, whether for sale or otherwise to anyone who is not an authorised user. 
This is the accepted version of the original article published by IEEE.

(C) 2019 IEEE. Personal use of this material is permitted. Permission from IEEE must be obtained for all other uses, in any current or future media, including reprinting/republishing this material for advertising or promotional purposes, creating new collective works, for resale or redistribution to servers or lists, or reuse of any copyrighted component of this work in other works. 


\title{
MULTICARRIER RADAR-COMMUNICATIONS WAVEFORM DESIGN FOR RF CONVERGENCE AND COEXISTENCE
}

\author{
Marian Bică and Visa Koivunen
}

Department of Signal Processing and Acoustics, Aalto University, Finland

\begin{abstract}
$\mathrm{RF}$ convergence where the same transceiver is used for communications and sensing purposes is taking place. In this paper, a dual-use radar-communications multicarrier waveform is proposed, where different subcarriers are assigned to different subsystems. Two algorithms for subcarrier assignment and optimal power allocation for the radar and communications subsystems are developed. A compound mutual information (MI) based objective function is used for optimizing the power allocation of each subsystem in both design algorithms. The first proposed design algorithm assumes priority for the radar subsystem and it is called radar selfish design. The second proposed design algorithm is called cooperative design, in which both subsystems are jointly optimized by maximizing a compound MI based objective function.
\end{abstract}

Index Terms - RF convergence, mutual information, waveform optimization, coexistence

\section{INTRODUCTION}

The area of coexistence among radar and communications systems has received plenty of attention from the research community [1-9]. A recent review on different approaches to the problems of coexistence, spectrum sharing and RF convergence available in the literature is given in [10]. It is expected that in the future RF convergence will be a key enabling technology in radar-communications coexistence. Different approaches to RF convergence have been proposed in the literature. For example, the tasks of the two subsystems are performed sequentially [11] or simultaneously, by leveraging different degrees of freedom in frequency [2], antenna elements [12] or radiation patterns $[13,14]$. Another approach is to embed communication symbols into the radar waveform as in [5-7,15]. Multicarrier waveforms are particularly suitable for RF convergence since most modern communications systems use these and they also bring many desirable properties for radar systems $[16,17]$.

In this paper a multicarrier waveform is assumed, for which interleaved subcarriers or subsets of subcarriers can be assigned to the radar or the communications tasks. Each subsystem is assigned distinct subsets of subcarriers from the total available subcarriers, as illustrated in Fig. 1. In a similar fashion, a spectrally interleaved OFDM system concept, with applications in radar network or MIMO radar, is proposed in [18]. Each transmitter would be allocated a subset of subcarriers such that overall each transmitter still uses the full bandwidth of the system. It is claimed in [18] that the blank subcarriers in the signal spectrum do not affect the radar sensing performance. A dual-use radar-communications waveform is developed in this paper for RF convergence. Mutual information (MI) has been employed for radar waveform optimization in several works, for example in [19-22]. Commonly, it is employed for target characterization or classification tasks, which could benefit form maximized

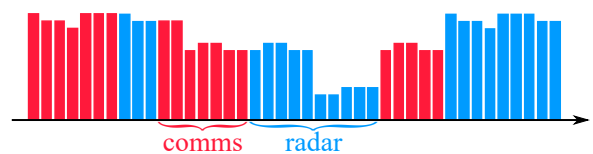

Fig. 1. Subcarrier assignment for radar or communications use.

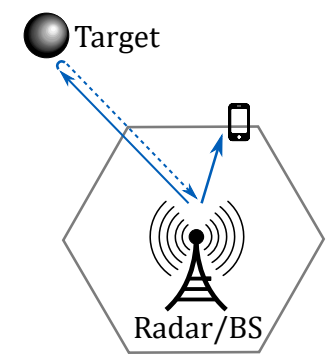

Fig. 2. Considered RF convergence system configuration.

MI between the received signal and target impulse response. In [23] based on [24] it is mentioned that, for a fixed probability of false alarm, MI maximization is related to the maximization of the probability of detection. Other optimization criteria such as probability of detection maximization [25] or CRB minimization [26] can be considered, however the optimization problems are no longer convex. In this paper a compound MI based objective function is formulated for subcarrier assignment and optimum power allocation to the radar and communications subsystems. Two algorithms for the design of the proposed dual-use waveform are also introduced, one called radar selfish design and one cooperative design. Also in [27] MI has been employed for a joint radar-communications system. However, therein all subcarriers are used for both tasks simultaneously and the objective function is formulated with respect to the maximized MI of the individual task.

Notation: A lower case bold letter $\mathrm{x}$ denotes a column vector, while a capital bold letter $\mathbf{X}$ denotes a matrix and $\mathbf{X}^{H}$ its Hermitian transpose. The $l$ th element in a vector is denoted $\mathbf{x}[l]$. By $I(\cdot)$ we denote the MI between two random variables and by $\|\mathbf{x}\|_{0}$ the $\ell_{0}$ norm - the number of non-zero entries of the vector $\mathbf{x}$.

\section{SYSTEM AND SIGNAL MODELS}

A joint radar-communications system intended for RF convergence is considered in this paper. The assumed coexistence and spectrum sharing co-located configuration is presented in Fig. 2. A multicarrier waveform, OFDM in this paper, is used by the system for both purposes. The dual-use radar-communications waveform is reflected back from the target, for radar purpose, and received at a communications receiver, for data transmission. 
Using the generalized multicarrier radar (GMR) model introduced in [28] and the special case for OFDM waveform with $N$ subcarriers, the dual-use waveform proposed in this paper can be modeled as follows:

$$
\mathbf{x}=\mathbf{F}^{H}[\mathbf{W r}+(\mathbf{I}-\mathbf{W}) \mathbf{c}]
$$

where vector $\mathbf{r}$ of size $N$ contains the frequency domain transmitted radar symbols and vector c of size $N$ contains the frequency domain transmitted communication symbols. The diagonal matrix $\mathbf{W}$ of size $N \times N$ is a subcarrier selection matrix whose elements take the values $\{0,1\}$, while matrix $\mathbf{F}^{H}$ is the inverse discrete Fourier transform (IDFT) matrix.

The transmitted waveform passes through one radar channel $h_{r}$, which contains the target, and one communications channel $h_{c}$, between the joint transciever and the communications receiver. The transmitted radar and communications symbols are assumed deterministic, while the radar and the communications channel impulse responses are assumed to be wide sense stationary (WSS) Gaussian processes with known second order statistics. These are similar assumptions as in $[20,29]$. Also, at both subsystem receivers, additive zero mean white Gaussian noise is assumed. Consequently, the signals at the radar and the communications receiver respectively can be written using a matrix formulation as follows:

$$
\left\{\begin{array}{l}
\mathbf{y}_{r}=\mathbf{X}_{r} \mathbf{h}_{r}+\mathbf{X}_{c} \mathbf{h}_{r}+\mathbf{n} \\
\mathbf{y}_{c}=\mathbf{X}_{r} \mathbf{h}_{c}+\mathbf{X}_{c} \mathbf{h}_{c}+\mathbf{m}
\end{array}\right.
$$

where $\mathbf{X}_{r}$ and $\mathbf{X}_{c}$ are Toeplitz matrices. These matrices can be approximated by circulant ones since their dimensions are sufficiently large [30]. Circulant matrices are diagonalized by unitary DFT matrices. The complex noise vectors $\mathbf{n}$ and $\mathbf{m}$, the complex radar channel taps $\mathbf{h}_{r}$ and communications channel taps $\mathbf{h}_{c}$ are all assumed zero mean Gaussian random vectors with known covariance matrices: $\sigma_{n}^{2} \mathbf{I}, \sigma_{m}^{2} \mathbf{I}, \mathbf{C}_{h_{r}}$ and $\mathbf{C}_{h_{c}}$ respectively.

\section{DUAL-USE SIGNAL DESIGN}

The chosen criteria for subcarrier selection is based on mutual information (MI). This criteria has been employed for both communications [31-33] and radar [19,20,22] waveform design. The radar subsystem wishes to maximize the MI between the received target reflected signal and the impulse response of the target. The communications subsystem wishes to maximize the MI between the received signal at the communications receiver and the transmitted signal. Consequently, a compound objective function is considered in this paper. The MI based compound objective function is formulated as follows:

$$
I\left(\mathbf{y}_{r} ; \mathbf{h}_{r}\right)+I\left(\mathbf{y}_{c} ; \mathbf{x}\right)
$$

which may be written as shown in our earlier work [20,29]:

$$
\begin{aligned}
& \frac{1}{2}\left[\sum_{k=0}^{N-1} \log \left(1+\frac{\mathbf{w}^{2}[k]|\mathbf{r}[k]|^{2} \boldsymbol{\sigma}_{h_{r}}^{2}[k]}{\mathbf{u}^{2}[k]|\mathbf{c}[k]|^{2} \boldsymbol{\sigma}_{h_{r}}^{2}[k]+\sigma_{n}^{2}}\right)\right. \\
& \left.+\sum_{k=0}^{N-1} \log \left(1+\frac{\mathbf{u}^{2}[k]|\mathbf{c}[k]|^{2} \boldsymbol{\sigma}_{h_{c}}^{2}[k]}{\mathbf{w}^{2}[k]|\mathbf{r}[k]|^{2} \boldsymbol{\sigma}_{h_{c}}^{2}[k]+\sigma_{m}^{2}}\right)\right],
\end{aligned}
$$

where $\boldsymbol{\sigma}_{h_{r}}^{2}[k]$ and $\boldsymbol{\sigma}_{h_{c}}^{2}[k]$ are the gains of the corresponding radar and communications channels on $k$ th subcarrier and $\sigma_{n}^{2}$ and $\sigma_{m}^{2}$ are the noise powers at the radar and communications receivers respectively. Also, $\mathbf{w}[k]$ and $\mathbf{u}[k]$ are the weights for the $k$ th subcarrier of the radar and communications subsystem respectively, given by the diagonal elements of matrices $\mathbf{W}$ and $\mathbf{U}=\mathbf{I}-\mathbf{W}$. These select the active or inactive subcarriers. As these take values only in $\{0,1\}$ and for any subcarrier $k$ only $\mathbf{w}[k]$ or $\mathbf{u}[k]$ is non-zero, the objective can be simplified to:

$$
\begin{aligned}
& \frac{1}{2}\left[\sum_{k=0}^{N-1} \log \left(1+\frac{\mathbf{w}[k]|\mathbf{r}[k]|^{2} \boldsymbol{\sigma}_{h_{r}}^{2}[k]}{\sigma_{n}^{2}}\right)\right. \\
& \left.+\sum_{k=0}^{N-1} \log \left(1+\frac{\mathbf{u}[k]|\mathbf{c}[k]|^{2} \boldsymbol{\sigma}_{h_{c}}^{2}[k]}{\sigma_{m}^{2}}\right)\right] .
\end{aligned}
$$

The objective function in (3) can be generalized and a weighting factor can be consider to each of the components.

Two dual-use waveform design strategies are proposed in the following. First, a radar selfish design is introduced, where the radar subsystem is designed first to achieve the best possible performance and may have access to all $N$ subcarriers. The number of subcarriers for the radar subsystem is then minimized in a second step while allowing for a controlled MI decrease. Subcarriers with the lowest channel gain will be unassigned from the radar subsystem and more power would be allocated to the ones with higher channel gain. The communications subsystem is then allocated also the spared subcarriers from the radar subsystem and it is optimized as well. Next, a cooperative design is developed, where subcarriers are first assigned to either subsystem such that the compound objective function in (5) is maximized. After that, each subsystem is optimized. A communications selfish design can also be employed similarly to the radar selfish one. However, this is not explored in this paper.

\subsection{Radar selfish design}

For this design strategy the radar waveform is first optimized based on MI maximization, similarly to [19-21,29]. At this stage, it is assumed that the weights $\mathbf{w}[k]$ are all 1 and the optimum power allocation for the radar subsystem is obtained based on MI maximization. As the optimization is performed for the radar subsystem only, the second sum in (5) can be ignored. Consequently, the optimization problem for the radar subsystem may be formulated as follows:

$$
\begin{array}{ll}
\underset{\left\{|\mathbf{r}[k]|^{2}\right\}}{\operatorname{maximize}} & \sum_{k=0}^{N-1} \log \left(1+\frac{\mathbf{w}[k]|\mathbf{r}[k]|^{2} \boldsymbol{\sigma}_{h_{r}}^{2}[k]}{\sigma_{n}^{2}}\right) \\
\text { subject to } & \sum_{k=0}^{N-1} \mathbf{w}[k]|\mathbf{r}[k]|^{2} \leq P_{\mathrm{T}} .
\end{array}
$$

After the optimal power allocation for the radar subsystem is obtained, the subcarriers which are allocated no power, due to very low channel quality, are assigned to the communications subsystem. Then, the optimization of the communications subsystem is formulated in a similar fashion, using the following optimization problem:

$$
\begin{array}{ll}
\underset{\left\{|\mathbf{c}[k]|^{2}\right\}}{\operatorname{maximize}} & \sum_{k=0}^{N-1} \log \left(1+\frac{\mathbf{u}[k]|\mathbf{c}[k]|^{2} \boldsymbol{\sigma}_{h_{c}}^{2}[k]}{\sigma_{m}^{2}}\right) \\
\text { subject to } & \sum_{k=0}^{N-1} \mathbf{u}[k]|\mathbf{c}[k]|^{2} \leq P_{\mathrm{T}} .
\end{array}
$$

It is observed from (6) and (7) that the same total power constraint is imposed to both subsystems. Both (6) and (7) can be solved exactly, for example as in [28], using their Lagrangian form and the KarushKuhn-Tucker (KKT) conditions [34]. The solutions to (6) and (7) are water filling solutions, where more power is allocated to subcarriers with higher channel gain and lower noise and interference power. Due to lack of space the exact power allocation solutions are not analytically derived here. An example of optimal power allocation is illustrated in Fig. 3. In the next step, the goal is to minimize the number of subcarriers assigned to the radar subsystem while controlling the reduction form the maximal MI. This will likely improve 


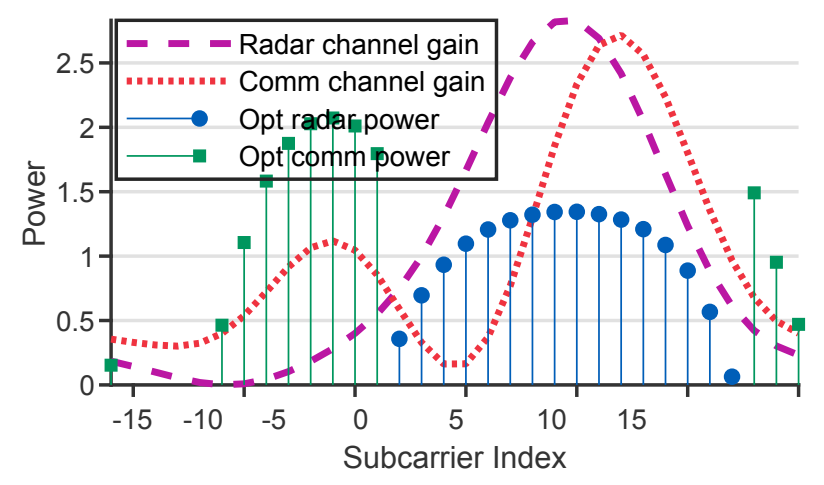

Fig. 3. Optimal power allocation for radar and communications subsystems. The radar system is optimized first and then the communications system using the spare subcarriers. Power is allocated to each subsystem to subcarriers with higher channel gain.

the performance of the communications subsystem. Consequently, the problem of minimizing the number of subcarriers assigned for the radar subsystem can be formulated as follows:

$$
\begin{array}{cl}
\underset{\{\mathbf{w}[k]\}}{\operatorname{minimize}} & \|\mathbf{w}\|_{0} \\
\text { subject to } & \sum_{k=0}^{N-1} \log \left(1+\frac{\mathbf{w}[k]|\mathbf{r}[k]|^{2} \boldsymbol{\sigma}_{h_{r}}^{2}[k]}{\sigma_{n}^{2}}\right) \geq t \\
& \mathbf{w}[k]=\{0,1\}, \forall k=0 \ldots N-1,
\end{array}
$$

where $|\mathbf{r}[k]|^{2}$ are the optimal radar powers obtained in the previous step from (6) and $t$ is a constraint on the minimum allowed radar MI. In this paper $t$ is considered $5-25 \%$ smaller than the maximum MI initially obtained from solving (6) with $\mathbf{w}=1$. This optimization problem is non-convex, however the best convex approximation can be used instead:

$$
\begin{array}{ll}
\underset{\{\mathbf{w}[k]\}}{\operatorname{minimize}} & \sum_{k=0}^{N-1} \mathbf{w}[k] \\
\text { subject to } & \sum_{k=0}^{N-1} \log \left(1+\frac{\mathbf{w}[k]|\mathbf{r}[k]|^{2} \boldsymbol{\sigma}_{h_{r}}^{2}[k]}{\sigma_{n}^{2}}\right) \geq t \\
& 0 \leq \mathbf{w}[k] \leq 1, \forall k=0 \ldots N-1,
\end{array}
$$

and the exact vector $\mathbf{w}$ can be obtained by rounding the solution for (9) to one of the values $\{0,1\}$. After the new vector $\mathbf{w}$ is obtained, the optimization problems (6) and (9) are solved again. This procedure is repeated until vector $\mathbf{w}$ does not change from one iteration to another. Next, the subcarriers assigned to the communications subsystems are obtained as $\mathbf{u}=\mathbf{1}-\mathbf{w}$ and the power allocation for the communications subsystem is optimized using (7). Algorithm 1 summarizes the radar selfish design strategy for the proposed dualuse waveform. An example of the final power allocation for both subsystems is illustrated in Fig. 4.

The trade-off between reduced maximum MI for radar and increased maximum MI for communications is evaluated next using simulations. For the communications subsystem the maximum MI is directly related to the capacity [35]. On the other hand, it may be difficult to quantify the performance loss in a radar task caused by a reduced maximum MI. Nevertheless, MI maximization is connected to minimum mean square error (MMSE), see [22,36]. Also, it was shown in [25] that a slight reduced detection performance is expected when waveforms and power allocation optimized based on MI are employed in a NP detector. It is shown in Fig. 5 how the maximum MI of each subsystem has changed from the first to the

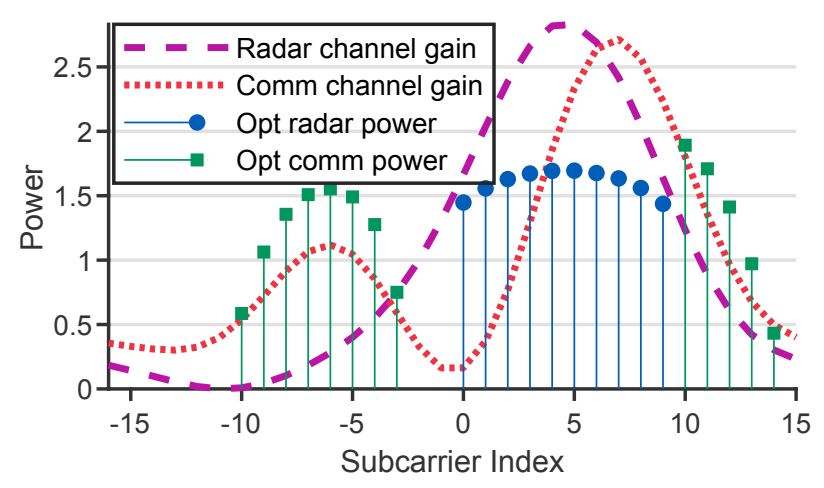

Fig. 4. Optimal power allocation for both subsystems after reducing the number of subcarriers for the radar subsystem.

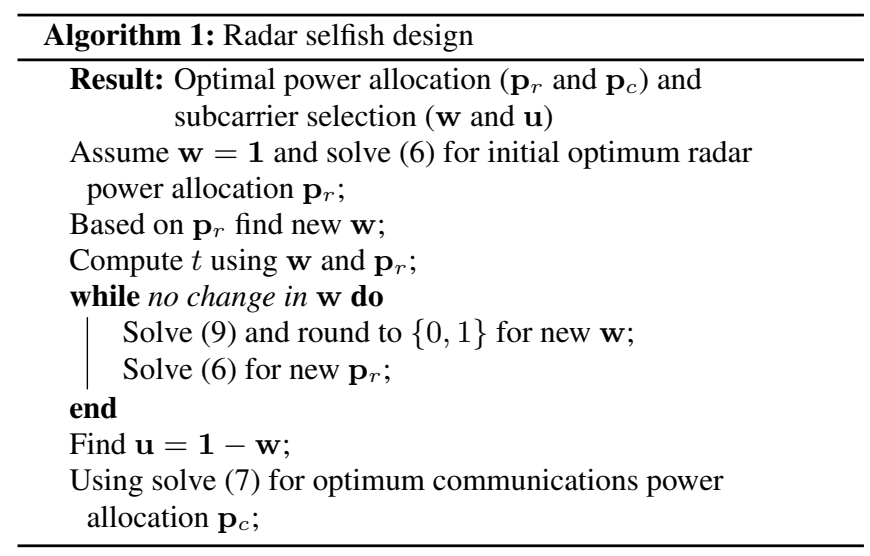

last step of Algorithm 1. The average maximum MI change is shown in Fig. 5 for 500 different channel realizations and a number of subcarriers $N=32,64,128$. It is observed that small decrease in radar maximum MI allows for a larger increase in communications maximum MI. Thus it can be concluded that it is worth taking a small loss in radar performance for a potential much larger gain for the communications subsystem. This result is consistent for different number of available subcarriers.

\subsection{Cooperative design}

For this design strategy the available subcarriers are assigned for the radar or the communications subsystem based on maximizing the compound objective in (5). Considering uniform power over the subcarriers for both radar and communications subsystems and the fact that for any given $k$ th subcarrier either $\mathbf{w}[k]$ or $\mathbf{u}[k]$ is non-zero, the compound objective function in (5) can be simplified to:

$$
\frac{1}{2}\left[\sum_{k=0}^{N-1} \log \left(1+\frac{\mathbf{w}[k] \boldsymbol{\sigma}_{h_{r}}^{2}[k]}{\sigma_{n}^{2}}+\frac{\mathbf{u}[k] \boldsymbol{\sigma}_{h_{c}}^{2}[k]}{\sigma_{m}^{2}}\right)\right] .
$$

It can be found that the optimum $\mathbf{w}[k]$ and $\mathbf{u}[k]$ which maximize the objective in (10) are given by:

$$
\begin{cases}\text { If } \frac{\boldsymbol{\sigma}_{h_{c}}^{2}[k]}{\sigma_{m}^{2}}>\frac{\boldsymbol{\sigma}_{h_{r}}^{2}[k]}{\sigma_{n}^{2}} & \text { then } \mathbf{w}[k]=0, \mathbf{u}[k]=1 \\ \text { If } \frac{\boldsymbol{\sigma}_{h_{c}}^{2}[k]}{\sigma_{m}^{2}} \leq \frac{\boldsymbol{\sigma}_{h_{r}}^{2}[k]}{\sigma_{n}^{2}} & \text { then } \mathbf{w}[k]=1, \mathbf{u}[k]=0 .\end{cases}
$$

After the vectors $\mathbf{w}$ and $\mathbf{u}$ are obtained, the optimal power allocation based on MI maximization is found for both the radar and the communications subsystems. An example of optimal power allocation 


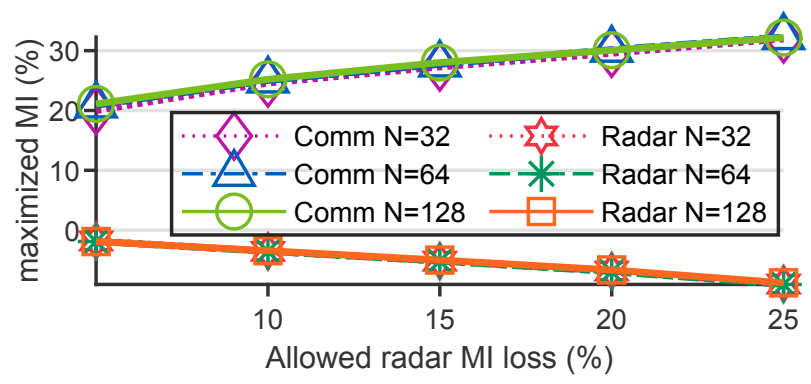

Fig. 5. The change in maximum MI for each subsystem. It pays off to allow a small decrease in radar maximum MI for a larger communications maximum MI - which translates to a higher capacity. This is valid for waveforms with $N=32,64,128$ subcarriers.

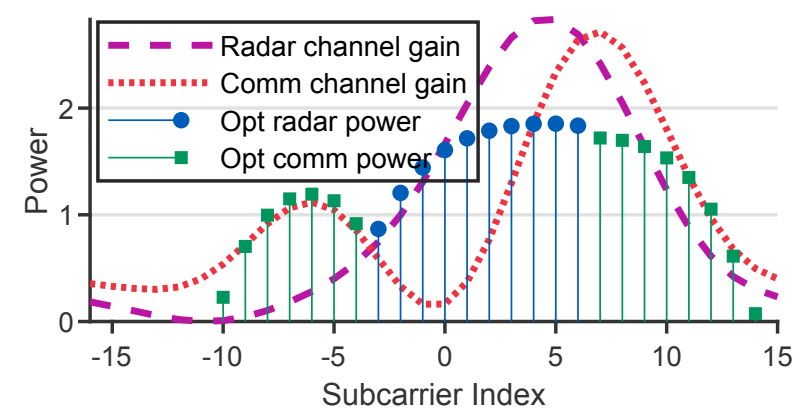

Fig. 6. Optimal power allocation for radar and communications subsystem when the cooperative design is employed.

for both radar and communications subsystems using this cooperative design is shown in Fig. 6.

\subsection{Radar selfish versus cooperative design}

We can quantify how much each subsystem gains in the cooperative design versus the radar selfish design. The final maximum MI for both subsystems obtained using the radar selfish and the cooperative design strategies is shown in Fig. 7. As expected, both subsystems achieve similar maximum MI using the cooperative design. It can be concluded from the results in Fig. 7 that the cooperative design is better for the communications subsystem given the choice of $5-25 \%$ loss in maximum radar MI. As expected, for the radar subsystem the radar selfish design is more favorable as long as there is not too much loss allowed for its maximum MI. For the simulation results presented in Fig. 7 this point is around $22 \%$ loss.

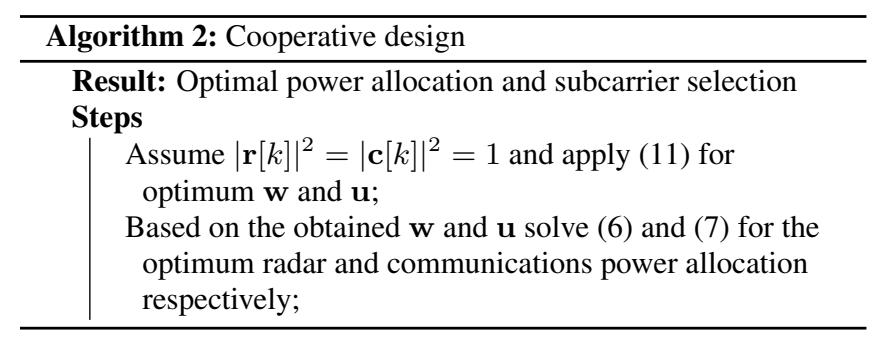

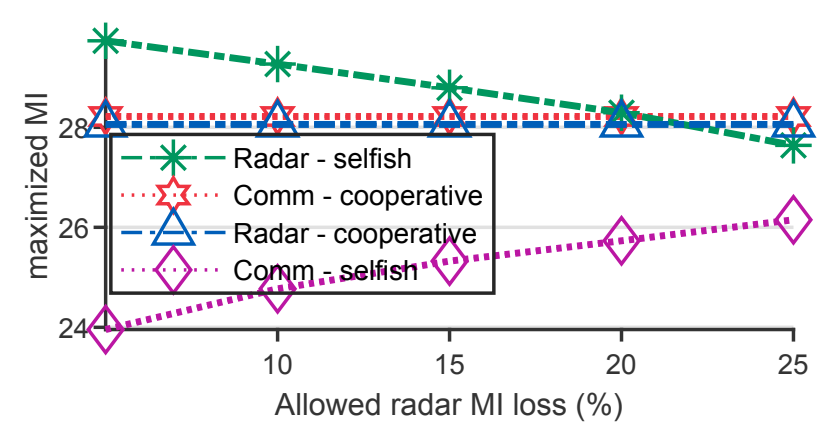

Fig. 7. Comparison between the proposed designs for waveforms with $N=128$ subcarriers. The cooperative design is better for the communications subsystem, while the radar selfish design is better for the radar subsystem as long as there is not too much loss allowed for its maximum MI.

\section{CONCLUSIONS}

A dual-function radar-communications OFDM waveform is proposed in this paper. Two design strategies for subcarrier assignment and optimal power allocation using MI based criteria are also proposed. The first design strategy is radar selfish, which means that the MI optimal performance for the radar is ensured first. After that, by allowing certain maximal MI loss in the radar subsystem, the number of subcarriers for the radar subsystem is minimized and the spare subcarriers are reassigned to the communications subsystem. The purpose of this is to allow for higher communications data rates. The second design strategy is cooperative, which means that subcarriers are assigned to either subsystem based on the channel gain experienced by the corresponding subcarriers. After that, the optimal power allocation for both subsystems is obtained using MI based criteria. It is shown using simulation results that the cooperative design is better for the communication subsystem, while the radar selfish design is better for the radar one as long as the loss in maximum MI is not too large.

\section{REFERENCES}

[1] A. Hassanien, M. G. Amin, Y. D. Zhang, and B. Himed, "A dual-function MIMO radar-communications system using PSK modulation," in 2016 24th European Signal Processing Conference (EUSIPCO), August 2016, pp. 1613-1617.

[2] A. Hassanien, B. Himed, and B. D. Rigling, "A dual-function MIMO radar-communications system using frequency-hopping waveforms," in 2017 IEEE Radar Conference (RadarConf), May 2017, pp. 1721-1725.

[3] E. Grossi, M. Lops, L. Venturino, and A. Zappone, "Opportunistic Radar in IEEE 802.11ad Networks," IEEE Transactions on Signal Processing, vol. 66, no. 9, pp. 2441-2454, May 2018.

[4] L. Zheng, M. Lops, X. Wang, and E. Grossi, "Joint Design of Overlaid Communication Systems and Pulsed Radars," IEEE Transactions on Signal Processing, vol. 66, no. 1, pp. 139-154, January 2018.

[5] S. D. Blunt, P. Yatham, and J. Stiles, "Intrapulse radarembedded communications," IEEE Transactions on Aerospace and Electronic Systems, vol. 46, no. 3, pp. 1185-1200, July 2010. 
[6] J. G. Metcalf, C. Sahin, S. D. Blunt, and M. Rangaswamy, "Analysis of symbol-design strategies for intrapulse radarembedded communications," IEEE Transactions on Aerospace and Electronic Systems, vol. 51, no. 4, pp. 2914-2931, Oct 2015.

[7] S. D. Blunt, J. G. Metcalf, C. R. Biggs, and E. Perrins, "Performance characteristics and metrics for intra-pulse radarembedded communication," IEEE Journal on Selected Areas in Communications, vol. 29, no. 10, pp. 2057-2066, December 2011.

[8] A. Hassanien, M. G. Amin, Y. D. Zhang, and F. Ahmad, "Signaling strategies for dual-function radar communications: an overview," IEEE Aerospace and Electronic Systems Magazine, vol. 31, no. 10, pp. 36-45, October 2016.

[9] A. Hassanien, M. G. Amin, Y. D. Zhang, and F. Ahmad, "Dualfunction radar-communications: Information embedding using sidelobe control and waveform diversity," IEEE Transactions on Signal Processing, vol. 64, no. 8, pp. 2168-2181, April 2016.

[10] B. Paul, A. R. Chiriyath, and D. W. Bliss, "Survey of RF Communications and Sensing Convergence Research," IEEE Access, vol. 5, pp. 252-270, December 2017.

[11] J. Moghaddasi and K. Wu, "Multifunctional Transceiver for Future Radar Sensing and Radio Communicating Data-Fusion Platform," IEEE Access, vol. 4, pp. 818-838, 2016.

[12] G. C. Tavik, C. L. Hilterbrick, J. B. Evins, J. J. Alter, J. G. Crnkovich, J. W. de Graaf, W. Habicht, G. P. Hrin, S. A. Lessin, D. C. Wu, and S. M. Hagewood, "The advanced multifunction rf concept," IEEE Transactions on Microwave Theory and Techniques, vol. 53, no. 3, pp. 1009-1020, March 2005.

[13] Z. Geng, R. Xu, H. Deng, and B. Himed, "Fusion of radar sensing and wireless communications by embedding communication signals into the radar transmit waveform," IET Radar, Sonar Navigation, vol. 12, no. 6, pp. 632-640, May 2018.

[14] A. Ahmed, Y. Gu, D. Silage, and Y. D. Zhang, "Powerefficient multi-user dual-function radar-communications," in 2018 IEEE 19th International Workshop on Signal Processing Advances in Wireless Communications (SPAWC), June 2018, pp. 1-5.

[15] C. Sahin, J. Jakabosky, P. M. McCormick, J. G. Metcalf, and S. D. Blunt, "A novel approach for embedding communication symbols into physical radar waveforms," in 2017 IEEE Radar Conference (RadarConf), May 2017, pp. 1498-1503.

[16] C. Sturm, T. Zwick, and W. Wiesbeck, "An OFDM System Concept for Joint Radar and Communications Operations," in Proceedings of 69th IEEE Vehicular Technology Conference, April 2009, pp. 1-5.

[17] C. Sturm and W. Wiesbeck, "Waveform Design and Signal Processing Aspects for Fusion of Wireless Communications and Radar Sensing," Proceedings of the IEEE, vol. 99, no. 7, pp. 1236-1259, July 2011.

[18] C. Sturm, Y. L. Sit, M. Braun, and T. Zwick, "Spectrally interleaved multi-carrier signals for radar network applications and multi-input multi-output radar," IET Radar, Sonar Navigation, vol. 7, no. 3, pp. 261-269, March 2013.

[19] M.R. Bell, "Information theory and radar waveform design," IEEE Transactions on Information Theory, vol. 39, no. 5, pp. 1578-1597, September 1993.
[20] K. W. Huang, M. Bica, U. Mitra, and V. Koivunen, "Radar Waveform Design in Spectrum Sharing Environment: Coexistence and Cognition," in 2015 IEEE Radar Conference (RadarCon), May 2015, pp. 1698-1703.

[21] R. A. Romero, J. Bae, and N. A. Goodman, "Theory and Application of SNR and Mutual Information Matched Illumination Waveforms," IEEE Transactions on Aerospace and Electronic Systems, vol. 47, no. 2, pp. 912-927, April 2011.

[22] Yang Yang and R.S. Blum, "MIMO radar waveform design based on mutual information and minimum mean-square error estimation," IEEE Transactions on Aerospace and Electronic Systems, vol. 43, no. 1, pp. 330-343, January 2007.

[23] A. De Maio, M. Lops, and L. Venturino, "Diversity-Integration Tradeoffs in MIMO Detection," IEEE Transactions on Signal Processing, vol. 56, no. 10, pp. 5051-5061, October 2008.

[24] A. D. Maio and M. Lops, "Design Principles of MIMO Radar Detectors," IEEE Transactions on Aerospace and Electronic Systems, vol. 43, no. 3, pp. 886-898, July 2007.

[25] M. Bica, K. W. Huang, U. Mitra, and V. Koivunen, "Opportunistic Radar Waveform Design in Joint Radar and Cellular Communication Systems," in 2015 IEEE Global Communications Conference (GLOBECOM), December 2015, pp. 1-7.

[26] M. Bică and V. Koivunen, "Radar Waveform Optimization for Target Parameter Estimation in Cooperative RadarCommunications Systems," IEEE Transactions on Aerospace and Electronic Systems, pp. 1-1, 2018, published December 5.

[27] Y. Liu, G. Liao, J. Xu, Z. Yang, and Y. Zhang, "Adaptive OFDM Integrated Radar and Communications Waveform Design Based on Information Theory," IEEE Communications Letters, vol. 21, no. 10, pp. 2174-2177, Oct 2017.

[28] M. Bică and V. Koivunen, "Generalized Multicarrier Radar: Models and Performance," IEEE Transactions on Signal Processing, vol. 64, no. 17, pp. 4389-4402, September 2016.

[29] M. Bica, K. W. Huang, V. Koivunen, and U. Mitra, "Mutual information based radar waveform design for joint radar and cellular communication systems," in 2016 IEEE International Conference on Acoustics, Speech and Signal Processing (ICASSP), March 2016, pp. 3671-3675.

[30] R.M. Gray, Toeplitz and Circulant Matrices: A Review, Foundations and Trends in Technology.

[31] A. Scaglione, S. Barbarossa, and G. B. Giannakis, "Filterbank transceivers optimizing information rate in block transmissions over dispersive channels," IEEE Transactions on Information Theory, vol. 45, no. 3, pp. 1019-1032, April 1999.

[32] G. G. Raleigh and J. M. Cioffi, "Spatio-temporal coding for wireless communication," IEEE Transactions on Communications, vol. 46, no. 3, pp. 357-366, March 1998.

[33] D. P. Palomar and J. R. Fonollosa, "Practical algorithms for a family of waterfilling solutions," IEEE Transactions on Signal Processing, vol. 53, no. 2, pp. 686-695, Feb 2005.

[34] Stephen Boyd and Lieven Vandenberghe, Convex Optimization, Cambridge University Press, New York, NY, USA, 2004.

[35] T.M. Cover and J.A. Thomas, Elements of Information Theory, A Wiley-Interscience publication. Wiley, 2006.

[36] D. Guo, S. Shamai, and S. Verdú, The Interplay Between Information and Estimation Measures, Foundations and trends in signal processing. 